\title{
Criminologie
}

\section{Éditorial : vers les applications pratiques de l'analyse spatiale du crime}

\section{Marc Ouimet}

Volume 27, numéro 1, 1994

Analyse spatiale du crime

URI : https://id.erudit.org/iderudit/017344ar

DOI : https://doi.org/10.7202/017344ar

Aller au sommaire du numéro

Éditeur(s)

Les Presses de l'Université de Montréal

ISSN

0316-0041 (imprimé)

1492-1367 (numérique)

Découvrir la revue

Citer ce document

Ouimet, M. (1994). Éditorial : vers les applications pratiques de l'analyse spatiale du crime. Criminologie, 27(1), 3-5. https://doi.org/10.7202/017344ar d'utilisation que vous pouvez consulter en ligne.

https://apropos.erudit.org/fr/usagers/politique-dutilisation/ 
Le crime, comme de nombreux autres phénomènes sociaux, $n$ 'est pas distribué aléatoirement dans l'espace. Par exemple, l'homicide est plus fréquent dans la région métropolitaine de Montréal que dans le reste du Québec. On peut aussi penser que certaines zones du territoire montréalais ont plus d'homicides que d'autres. Finalement, à l'intérieur de zones où l'homicide est plus fréquent qu'ailleurs, certains segments de rue sont plus dangereux que d'autres. Ces données montrent que le crime est spatialement déterminé et donnent raison à ceux qui considèrent les risques de victimisation estimés avant de planifier leurs déplacements quotidiens.

Depuis ses débuts, l'analyse spatiale en criminologie a pris une variété de formes. Le statisticien belge Quételet a établi la distribution spatiale des crimes en France et l'a comparée à d'autres statistiques morales (1831). Guerry était fasciné par la constance des nombres du crime. Selon lui : «Non seulement les crimes sont commis dans une proportion connue, en un lieu déterminé, par des individus dont le sexe et l'âge sont prévus, une saison est encore affectée à chacun d'eux » (1833, p. 11). Plus tard, Park et Burgess (1925), de même que Shaw et McKay (1942), identifièrent les zones les plus criminogènes de Chicago. Plus récemment, l'américain Lebeau (1992) a montré que les agresseurs sexuels suivaient des patterns spatiaux-temporels spécifiques. Compte tenu de la diversité des thèmes développés et des méthodes de recherche suivies par ceux qui ont intégré l'espace à leur propos, il n'y a pas, à proprement parler, une tradition de recherche en analyse spatiale du crime.

En utilisant la catégorisation des niveaux d'analyse en criminologie développée par Pinatel (1963), il est possible de regrouper les pratiques des chercheurs qui ont intégré l'espace dans leur conceptualisation. Premièrement, l'analyse spatiale peut éclairer le chercheur qui étudie l'incidence de la criminalité. Par exemple, on tentera d'identifier les facteurs sociodémographiques pouvant expliquer la distribution de l'incidence de la criminalité observée pour différents quartiers d'une ville. Deuxièmement, l'analyse spatiale est utile à celui ou celle qui étudie le crime, c'est-à-dire, qui cherche à comprendre les éléments expliquant l'occurrence d'un crime à un endroit et à un moment précis. On pourrait, par exemple, identifier précisément sur la carte d'un quartier l'endroit où un certain nombre de vols de véhicules à moteur ont eu lieu et tenter d'expliquer la distribution

1. Professeur, École de criminologie, Université de Montréal, C.P. 6128 , Succ. A, Montréal, Québec, H3C 337. 
observée (par exemple, y a-t-il une voie d'accès à une autoroute à proximité des concentrations?). Troisièmement, des études montrant comment des délinquants spécialisés (par exemple, des braqueurs de banque, des pyromanes, des pédophiles, etc.) circulent dans l'espace, entre leur lieu de résidence et l'endroit où ils commettent leur délit, permettraient de faire avancer les connaissances de la criminologie sur les criminels.

Le principal défi pour l'analyse spatiale reste encore le développement de modèles théoriques servant à expliquer les distributions spatiales observables de la criminalité. En effet, les cartes de la distribution spatiale du crime dans l'espace ne nous apprennent que peu de choses sur les phénomènes observés ; leur intérêt théorique est plutôt limité. Par contre, c'est au plan des applications pratiques que la dimension spatiale du crime devient vraiment intéressante. L'endroit où le crime est commis apparaît quelquefois comme la seule piste de recherche qui pourra mener policiers et enquêteurs à identifier l'agresseur (par exemple, dans le cas de la disparition d'un enfant).

Les développements récents et ceux à venir dans le monde de l'informatique auront certainement un impact important sur plusieurs aspects du problème criminel. De nouvelles banques de données portant sur les crimes commis et sur les criminels connus permettront de faire progresser le travail de prévention et d'enquête policière si celles-ci sont intégrées à des systèmes experts de nature géomatique. Par exemple, Block (1992) a mis au point un système d'analyse spatiale de la criminalité à Chicago qui vise la détection des concentrations anormalement élevées de crimes dans l'espace et qui permet de diriger l'action préventive de la police dans des secteurs précis de la ville.

Mais avant de passer à des applications efficaces de systèmes géomatiques, il faudra investir dans la recherche fondamentale sur des thèmes aussi variés que l'analyse des réseaux criminels, l'analyse des carrières criminelles, l'analyse du cheminement des délinquants pathologiques, etc. Ce dossier de la revue sur l'analyse spatiale n'est qu'un premier pas dans cette direction. 


\section{BIBLIOGRAPHIE}

BLOCK, Carolyn R. (1992), Computer Mapping As a Tool in Violence Reduction, Séminaire d'analyse criminologique, École de criminologie, Université de Montréal.

GUERRY, A.M. (1833), Essai sur la statistique morale en France, Paris, Crochart.

LEBEAU, J. (1992), « Four Cases Studies Illustrating the Spatial-Temporal Analysis of Serial Rapists «, Police Studies, vol. 15, n 3, p. 124-145.

PARK, R.E. et E.W. BURGESS (1925), The City. Chicago, University of Chicago Press.

PINATEL, J. (1963), Criminologie, Paris, Dalloz.

QUÉTELET, A. (1831), Recherches sur le penchant au crime aux différents âges, Mémoire présenté à l'Académie Royale des sciences.

SHAW, C. et H. MCKAY (1942), Juvenile Delinquency in Urban Areas, Chicago, University of Chicago Press. 